ANNALES

POLONICI MATHEMATICI

LXXIX.1 (2002)

\title{
Invariant measures related with randomly connected Poisson driven differential equations
}

\author{
by Katarzyna Horbacz (Katowice)
}

Abstract. We consider the stochastic differential equation

$$
d u(t)=a(u(t), \xi(t)) d t+\int_{\Theta} \sigma(u(t), \theta) \mathcal{N}_{p}(d t, d \theta) \quad \text { for } t \geq 0
$$

with the initial condition $u(0)=x_{0}$. We give sufficient conditions for the existence of an invariant measure for the semigroup $\left\{P^{t}\right\}_{t \geq 0}$ corresponding to (1). We show that the existence of an invariant measure for a Markov operator $P$ corresponding to the change of measures from jump to jump implies the existence of an invariant measure for the semigroup $\left\{P^{t}\right\}_{t \geq 0}$ describing the evolution of measures along trajectories and vice versa.

0. Introduction. We will consider the stochastic differential equation

$$
d u(t)=a(u(t), \xi(t)) d t+\int_{\Theta} \sigma(u(t), \theta) \mathcal{N}_{p}(d t, d \theta) \quad \text { for } t \geq 0
$$

with the initial condition

$$
u(0)=x_{0},
$$

where $\{u(t)\}_{t \geq 0}$ is a stochastic process with values in $\mathbb{R}^{m}$ and $\{\xi(t)\}_{t \geq 0}$ is a stochastic process with values in $\{1, \ldots, N\}$ which describes random switching at random moments $t_{n}$. The precise assumptions concerning the coefficients $a: \mathbb{R}^{m} \times\{1, \ldots, N\} \rightarrow \mathbb{R}^{m}$ and $\sigma: \mathbb{R}^{m} \times \Theta \rightarrow \mathbb{R}^{m}$ and the Poisson random measure $\mathcal{N}_{p}$ will be formulated in Section 2 .

Our main aim is to study the problem of the existence of an invariant measure for the Markov semigroup $\left\{P^{t}\right\}_{t \geq 0}$ associated with equation (0.1).

We reduce the problem to a similar problem for a simpler Markov chain $\left\{x_{n}\right\}_{n \geq 1}$, where

$$
x_{n}=u\left(t_{n}\right) \quad \text { for } n=1,2, \ldots
$$

2000 Mathematics Subject Classification: Primary 60H10, 34F05; Secondary 60J25, $37 \mathrm{C} 45$.

Key words and phrases: Markov semigroup, invariant measure, stochastic differential equation. 
and $t_{n}$ are the jump times. Then there exists an operator $P$ corresponding to the change of measures from jump to jump.

We show that the semigroup $\left\{P^{t}\right\}_{t \geq 0}$ has an invariant measure if and only if the operator $P$ has the same property.

The similar problem for a simpler equation was considered in [4].

In the case when the coefficient $a: \mathbb{R}^{m} \times\{1, \ldots, N\} \rightarrow \mathbb{R}^{m}$ does not depend on the second variable, that is, the differential equations do not randomly switch at random jump moments, we obtain the stochastic equation considered by A. Lasota and J. Traple [10].

We also discuss the relations between the generalized Rényi dimension (the $L^{\infty}$-dimension) of the invariant measure of $\left\{P^{t}\right\}_{t \geq 0}$ if the dimension of the invariant measure of $P$ is known and vice versa.

Finally observe that there are numerous phenomena that can be reasonally modeled as a response to the points of a marked point process. For instance in [14] there are many examples of problems which generate such equations. Recently equations of type (0.1) have appeared in a model of the growth of a size-structured population of cells [1].

The organization of the paper is as follows: Section 1 contains some notation and definitions from the theory of Markov operators. In Section 2 we specify the problem to be considered. The relationship between the transition operator and the semigroup generated by $(0.1)$ is formulated in Section 3. In Section 4 we give sufficient conditions for the existence of an invariant measure for the transition operator. In Section 5 we find the relations between the generalized Rényi dimensions of the invariant measure of $\left\{P^{t}\right\}_{t \geq 0}$ and $P$.

1. Preliminaries. Let $(Y, \varrho)$ be a separable metric space. Throughout this paper we assume that $K(x, r)$ stands for the closed ball in $Y$ with center at $x$ and radius $r$. We denote by $\mathcal{B}(Y)$ the $\sigma$-algebra of Borel subsets of $Y$, by $\mathcal{M}(Y)$ the family of all finite Borel measures (nonnegative, $\sigma$-additive) on $Y$, and by $\mathcal{M}_{1}(Y)$ the subset of those $\mu \in \mathcal{M}(Y)$ such that $\mu(Y)=1$. The elements of $\mathcal{M}_{1}(Y)$ will be called distributions. Further

$$
\mathcal{M}_{\text {sig }}(Y)=\left\{\mu_{1}-\mu_{2}: \mu_{1}, \mu_{2} \in \mathcal{M}(Y)\right\}
$$

is the space of finite signed measures.

As usual, $B(Y)$ denotes the space of all bounded Borel measurable functions $f: Y \rightarrow \mathbb{R}, C(Y)$ the subspace of all bounded continuous functions with the supremum norm $\|\cdot\|_{C}$, and $C_{0}(Y)$ the subspace of functions with compact support $C(Y)$.

$C_{0}^{(1)}\left(\mathbb{R}^{m} \times\{1, \ldots, N\}\right)$ is the subspace of $C_{0}\left(\mathbb{R}^{m} \times\{1, \ldots, N\}\right)$ of all functions with continuous and bounded first derivatives. For $f \in C_{0}^{(1)}\left(\mathbb{R}^{m} \times\right.$ $\{1, \ldots, N\})$ we denote by $f_{x}$ the gradient of the function $x \mapsto f(x, k)$. 
For $f \in B(Y)$ and $\mu \in \mathcal{M}_{\text {sig }}(Y)$ we write

$$
\langle f, \mu\rangle=\int_{Y} f(x) \mu(d x) .
$$

A linear mapping $P: \mathcal{M}_{\text {sig }}(Y) \rightarrow \mathcal{M}_{\text {sig }}(Y)$ is called a Markov operator if $P\left(\mathcal{M}_{1}(Y)\right) \subset \mathcal{M}_{1}(Y)$. Thus, for every distribution $\mu$ the measure $P \mu$ is also a distribution.

A measure $\mu_{*} \in \mathcal{M}(Y)$ is called invariant or stationary with respect to a Markov operator $P$ if $P \mu_{*}=\mu_{*}$. A stationary probabilistic measure is called a stationary distribution.

A Markov operator $P$ is called a Feller operator if there is an operator $U: B(Y) \rightarrow B(Y)($ dual to $P)$ such that

$$
\begin{gathered}
\langle U f, \mu\rangle=\langle f, P \mu\rangle \quad \text { for } f \in B(Y), \mu \in \mathcal{M}_{\text {sig }}(Y), \\
U f \in C(Y) \text { for } f \in C(Y) .
\end{gathered}
$$

Setting $\mu=\delta_{x}$ in (1.1) we obtain

$$
U f(x)=\left\langle f, P \delta_{x}\right\rangle \quad \text { for } f \in B(Y), x \in Y
$$

where $\delta_{x} \in \mathcal{M}_{1}(Y)$ is the point (Dirac) measure at $x$.

From (1.3) it follows immediately that $U$ is a linear operator satisfying

$$
\begin{array}{cl}
U f \geq 0 \quad \text { for } f \in B(Y), f \geq 0, \\
& U 1_{Y}=1_{Y} .
\end{array}
$$

Further, applying the Lebesgue monotone convergence theorem to the integral $\left\langle f, P \delta_{x}\right\rangle$, we obtain the implication

$$
\left.\begin{array}{l}
f_{n} \in B(Y) \\
f_{n+1} \leq f_{n} \\
\lim _{n \rightarrow \infty} f_{n}(x)=0
\end{array}\right\} \Rightarrow \lim _{n \rightarrow \infty} U f_{n}(x)=0 .
$$

Conditions (1.4)-(1.6) are quite important. They allow one to reverse the roles of $P$ and $U$. Namely assume that a linear operator $U: B(Y) \rightarrow B(Y)$ satisfying (1.4)-(1.6) is given. Then we may define $P: \mathcal{M}_{\text {sig }}(Y) \rightarrow \mathcal{M}_{\text {sig }}(Y)$ by setting

$$
P \mu(A)=\left\langle U 1_{A}, \mu\right\rangle \quad \text { for } \mu \in \mathcal{M}_{\text {sig }}(Y), A \in \mathcal{B}(Y) .
$$

It is easy to show that $P$ satisfies (1.1). Moreover, if $U$ satisfies (1.2) then $P$ is a Markov operator.

A family $\left\{P^{t}\right\}_{t \geq 0}$ of Markov operators is called a semigroup if $P^{t+s}=$ $P^{t} \circ P^{s}$ for $t, s \in \mathbb{R}_{+}$and $P^{0}=I$ is the identity operator on $\mathcal{M}_{1}(Y)$. If the operators $P^{t}, t \geq 0$, are Feller, we say that $\left\{P^{t}\right\}_{t \geq 0}$ is a Feller semigroup. $\left\{T^{t}\right\}_{t \geq 0}$ denotes the semigroup dual to $\left\{P^{t}\right\}_{t \geq 0}$, i.e.

$$
\left\langle T^{t} f, \mu\right\rangle=\left\langle f, P^{t} \mu\right\rangle \quad \text { for } f \in C(Y), \mu \in \mathcal{M}_{1}(Y) .
$$


Given $\mu \in \mathcal{M}_{1}(Y)$ we define

$$
\begin{aligned}
& \overline{\operatorname{dim}}_{\infty} \mu=\limsup _{r \rightarrow 0} \frac{\log (\sup \{\mu(K(x, r)): x \in Y\})}{\log r}, \\
& \underline{\operatorname{dim}}_{\infty} \mu=\liminf _{r \rightarrow 0} \frac{\log (\sup \{\mu(K(x, r)): x \in Y\})}{\log r} .
\end{aligned}
$$

If $\overline{\operatorname{dim}}_{\infty} \mu=\underline{\operatorname{dim}}_{\infty} \mu$, then the common value is called the generalized Rényi dimension (the $L^{\infty}$-dimension) of $\mu$ and it is denoted by $\operatorname{dim}_{\infty} \mu$ ([12], [13]). In many papers $\operatorname{dim}_{\infty} \mu$ is called the Lévy concentration dimension of $\mu$ ([9], [10]).

2. Formulation of the problem. We consider the differential equation with Poisson type perturbations

$$
d u(t)=a(u(t), \xi(t)) d t+\int_{\Theta} \sigma(u(t), \theta) \mathcal{N}_{p}(d t, d \theta) \quad \text { for } t \geq 0
$$

with the initial condition

$$
u(0)=x_{0}
$$

We make the following assumptions:

(i) The coefficient $a: \mathbb{R}^{m} \times\{1, \ldots, N\} \rightarrow \mathbb{R}^{m}$ is Lipschitzian with respect to the $\mathbb{R}^{m}$ variable:

$$
\|a(x, k)-a(y, k)\| \leq L\|x-y\| \quad \text { for }(x, k),(y, k) \in \mathbb{R}^{m} \times\{1, \ldots, N\} .
$$

(ii) There are given a probability space $(\Omega, \mathcal{F}$, prob) and a sequence $\left\{t_{n}\right\}_{n \in \mathbb{N}}$ of random variables such that the variables $\Delta t_{i}=t_{i}-t_{i-1}\left(t_{0}=0\right)$ are nonnegative, independent and equally distributed with density $g(t)=$ $\lambda e^{-\lambda t}$ for $t \geq 0$.

(iii) Let $(\Theta, \mathcal{G}, \nu)$ be a finite measure space with $\nu(\Theta)=1$. Let $\left\{\theta_{i}\right\}_{i \in \mathbb{N}}$ be a sequence of random elements with values in $\Theta$. The elements $\theta_{i}$ are independent and equally distributed with distribution $\nu$. The sequences $\left\{t_{i}\right\}_{i \in \mathbb{N}}$ and $\left\{\theta_{i}\right\}_{i \in \mathbb{N}}$ are independent.

(iv) Moreover, suppose we are given a probability matrix $\left[p_{i j}(x)\right]_{i, j=1}^{N}$ such that

$$
p_{i j}(x) \geq 0, \quad \sum_{j=1}^{N} p_{i j}(x)=1 \quad \text { for } x \in \mathbb{R}^{m} \text { and } i, j=1, \ldots, N .
$$

Denote by $v_{k}(t)=\Pi_{k}(t, x)$ the solution of the unperturbed Cauchy problem

$$
d v_{k}(t)=a\left(v_{k}(t), k\right) d t, \quad v_{k}(0)=x, \quad x \in \mathbb{R}^{m}, k=1, \ldots, N .
$$

Set

$$
q(x, \theta)=x+\sigma(x, \theta) \quad \text { for } x \in \mathbb{R}^{m}, \theta \in \Theta .
$$


We consider a sequence of random variables $x_{n}: \Omega \rightarrow \mathbb{R}^{m}$ and a stochastic process $\xi(t): \Omega \rightarrow\{1, \ldots, N\}$ related by

$$
\begin{aligned}
& \xi(0)=k, \\
& \xi(t)=\xi\left(t_{n}\right) \quad \text { for } t_{n} \leq t<t_{n+1}, \\
& x_{n}=q\left(\Pi_{\xi\left(t_{n-1}\right)}\left(t_{n}-t_{n-1}, x_{n-1}\right), \theta_{n}\right),
\end{aligned}
$$

and

$$
\operatorname{prob}\left\{\xi\left(t_{n}\right)=s \mid x_{n}=y \text { and } x_{n}=q\left(\Pi_{k}\left(t_{n}-t_{n-1}, x_{n-1}\right), \theta_{n}\right)\right\}=p_{k s}(y),
$$

for $n=1, \ldots, N$.

(v) Let $q: \mathbb{R}^{m} \times \Theta \rightarrow \mathbb{R}^{m}$ be a measurable function such that $q(x, \cdot) \in$ $L^{1}(\nu)$ and

$$
\|q(x, \cdot)-q(y, \cdot)\|_{L^{1}(\nu)} \leq L_{q}\|x-y\| \quad \text { for all } x, y \in \mathbb{R}^{m}
$$

for some constant $L_{q} \geq 0$.

Conditions (iii) and (iv) imply that for each measurable set $Z \subset(0, \infty) \times \Theta$ the variable

$$
\mathcal{N}_{p}(Z)=\left\{i:\left(t_{i}, \theta_{i}\right) \in Z\right\}
$$

is Poisson distributed. It is called the Poisson random counting measure.

By a solution of $(2.1),(2.2)$ we mean a proces $\{X(t)\}_{t>0}$ with values in $\mathbb{R}^{m}$ such that with probability one the following two conditions are satisfied:

(a) The sample path is a right-continuous function such that for $t>0$ the limit $X(t-)=\lim _{s \rightarrow t^{-}} X(s)$ exists and

(b) $X(t)=x_{0}+\int_{0}^{t} a(x(s), \xi(s)) d s+\int_{0}^{t} \int_{\Theta} \sigma(\xi(s-), \theta) \mathcal{N}_{p}(d s, d \theta)$ for $t \geq 0$.

Observe that the solution $X(t)$ on each interval $\left[t_{k}, t_{k+1}\right), k=0,1, \ldots$, satisfies one of the ordinary differential equations

$$
d v_{s}(t)=a\left(v_{s}(t), s\right) d t, \quad t \in \mathbb{R}, s=1, \ldots, N,
$$

and the initial condition

$$
v_{s}\left(t_{k}\right)=X\left(t_{k}-\right)+\sigma\left(X\left(t_{k}-\right), \theta_{k}\right) .
$$

We can give an explicit formula for the solution $X(t): \Omega \rightarrow \mathbb{R}^{m}$ of (2.1), (2.2):

$$
\begin{aligned}
X(t) & =\Pi_{\xi\left(t_{n-1}\right)}\left(t-t_{n-1}, x_{n-1}\right) \quad \text { for } t_{n-1}<t<t_{n}, \\
X\left(t_{n}\right) & =x_{n} .
\end{aligned}
$$

We now recall the definition of the semigroup of Markov operators generated by the family of solutions of (2.1).

Let $Y=\mathbb{R}^{m} \times\{1, \ldots, N\}$ be equipped with the metric $\varrho$ given by

$$
\varrho((x, i),(y, j))=\|x-y\|+\varrho_{0}(i, j) \quad \text { for } x, y \in \mathbb{R}^{m} \text { and } i, j \in\{1, \ldots, N\}
$$


where

$$
\varrho_{0}(i, j)= \begin{cases}b & \text { for } i \neq j, \\ 0 & \text { for } i=j,\end{cases}
$$

and $b>0$ is some constant. For $x \in \mathbb{R}^{m}$ and $k \in\{1, \ldots, N\}$ denote by $(X(t), \xi(t))_{(x, k)}$ the solution of the initial value problem (2.1), (2.2). The process $(X(t), \xi(t))_{(x, k)}$ generates a semigroup $\left\{T^{t}\right\}_{t \geq 0}$ defined by

$$
T^{t} f(x, k)=E\left(f\left((X(t), \xi(t))_{(x, k)}\right)\right) \quad \text { for } f \in C(Y),
$$

where $E\left(f\left((X(t), \xi(t))_{(x, k)}\right)\right)$ denotes the mean value of $f\left((X(t), \xi(t))_{(x, k)}\right)$.

It is well known that $\left\{T^{t}\right\}_{t \geq 0}$ is a semigroup of operators from $C(Y)$ into itself and for every $t>0$ the operator $T^{t}$ is a contraction, i.e. $\left\|T^{t} f\right\|_{C} \leq$ $\|f\|_{C}$.

Now we define $P^{t}: \mathcal{M}_{1}(Y) \rightarrow \mathcal{M}_{1}(Y)$ by

$$
\left\langle P^{t} \mu, f\right\rangle=\left\langle\mu, T^{t} f\right\rangle \quad \text { for } f \in C(Y) \text { and } \mu \in \mathcal{M}_{1}(Y) \text {. }
$$

Setting

$$
G(t,(x, k), A)=\operatorname{prob}\left\{(X(t), \xi(t))_{(x, k)} \in A\right\}
$$

we obtain

$$
P^{t} \mu(A)=\int_{Y} G(t,(x, k), A) d \mu(x, k) \quad \text { for } \mu \in \mathcal{M}_{1}(Y) \text { and } A \in \mathcal{B}(Y) .
$$

The semigroup $\left\{P^{t}\right\}_{t \geq 0}$ is called the Markov semigroup generated by the problem (2.1), (2.2).

Moreover using (2.7) the semigroup $\left\{P^{t}\right\}_{t \geq 0}$ can be easily extended to the vector space $\mathcal{M}_{\text {sig }}(Y)$. For the first switching point $t_{1}$ there is a constant $k_{1}$ such that

$$
\begin{aligned}
\operatorname{prob}\left\{(X(h), \xi(h))_{(x, k)}=\left(\Pi_{\xi\left(t_{1}\right)}(h-\right.\right. & \left.\left.t_{1}, q\left(\Pi_{k}\left(t_{1}, x\right), \theta_{1}\right)\right), \xi\left(t_{1}\right)\right) 1_{[0, h]}\left(t_{1}\right) \\
+ & \left.\left(\Pi_{k}(h, x), k\right) 1_{[h,+\infty)}\left(t_{1}\right)\right\} \geq 1-k_{1} h^{2} .
\end{aligned}
$$

Since $f \in C(Y)$ is bounded and $t_{1}$ has density distribution function $\lambda e^{-\lambda t}$, we obtain

$$
\begin{aligned}
T^{h} f(x, k)= & \int_{\Theta} \int_{0}^{h} \sum_{s=1}^{N} f\left(\Pi_{s}\left(h-t, q\left(\Pi_{k}(t, x), \theta\right)\right), s\right) \\
& \times p_{k s}\left(q\left(\Pi_{k}(t, x), \theta\right)\right) \lambda e^{-\lambda t} d t d \nu(\theta) \\
& +f\left(\Pi_{k}(h, x), k\right) e^{-\lambda t}+\varepsilon_{1}(h)
\end{aligned}
$$

where $\left|\varepsilon_{1}(h)\right| \leq\|f\|_{C} k_{1} h^{2}$.

We will reduce the problem of the existence of an invariant measure for the semigroup $\left\{P^{t}\right\}_{t \geq 0}$ to the problem of the existence of an invariant measure for the Markov operator $P$ corresponding to the change of measures from jump to jump. 
Since

$$
X\left(t_{n}\right)=x_{n}=q\left(\Pi_{\xi\left(t_{n-1}\right)}\left(t_{n}-t_{n-1}, x_{n-1}\right), \theta_{n}\right) \quad \text { for } n \in \mathbb{N},
$$

setting

$\mu_{n}(A \times\{s\})=\operatorname{prob}\left\{x_{n} \in A\right.$ and $\left.x_{n}=q\left(\Pi_{s}\left(\Delta t_{n}, x_{n-1}\right), \theta_{n}\right)\right\}, \quad n=1,2, \ldots$, we obtain

where

$$
\mu_{n+1}=P \mu_{n}
$$

$$
P \mu(A)=\sum_{s=1}^{N} \int_{\Theta} \int_{Y} \int_{0}^{\infty} 1_{A}\left(q\left(\Pi_{s}(t, x), \theta\right), s\right) \lambda e^{-\lambda t} p_{k s}(x) d t d \nu(\theta) d \mu(x, k)
$$

for $\mu \in \mathcal{M}(Y)$ and $A \in \mathcal{B}(Y)$. Analogously for every $f \in C(Y)$ we may calculate the conditional mean value of $f\left(X\left(t_{n+1}\right), \xi\left(t_{n+1}\right)\right)$ with respect to $\left(X\left(t_{n}\right), \xi\left(t_{n}\right)\right)$. Namely

$$
E\left(f\left(X\left(t_{n}\right), \xi\left(t_{n}\right)\right) \mid X\left(t_{n-1}\right)=x, \xi\left(t_{n-1}\right)=k\right)=U f(x, k)
$$

where

$$
U f(x, k)=\sum_{s=1}^{N} \int_{0}^{+\infty} \int_{\Theta} f\left(q\left(\Pi_{s}(t, x), \theta\right), s\right) \lambda e^{-\lambda t} p_{k s}(x) d t d \nu(\theta)
$$

for $f \in C(Y),(x, k) \in Y$. From (2.8) and (2.9) it follows that $\langle f, P \mu\rangle=$ $\langle U f, \mu\rangle$ for $f \in C(Y), \mu \in \mathcal{M}_{1}(Y)$. Thus the adjoint operator $U^{*}$ is equal to $P$ on $\mathcal{M}_{1}(Y)$.

3. Main results. In order to formulate the main result of our paper we introduce two operators $H, G: \mathcal{M}_{1}(Y) \rightarrow \mathcal{M}_{1}(Y)$ by

$$
\begin{aligned}
& H \mu(A)=\int_{Y} \int_{\Theta} 1_{A}(q(x, \theta), k) d \nu(\theta) d \mu(x, k), \\
& G \mu(A)=\sum_{s=1}^{N} \int_{Y} \int_{0}^{\infty} 1_{A}\left(\Pi_{s}(t, x), s\right) p_{k s}(x) \lambda e^{-\lambda t} d t d \mu(x, k)
\end{aligned}
$$

for $A \in \mathcal{B}(Y)$. The following theorem shows the one-to-one correspondence between the set of $P$-invariant measures and the set of invariant measures with respect to $\left\{P^{t}\right\}_{t \geq 0}$.

THEOREM 3.1. If $\mu_{0} \in \mathcal{M}_{1}(Y)$ satisfies the condition

$$
P \mu_{0}=\mu_{0}
$$

and if $\widetilde{\mu}=G \mu_{0}$, then

$$
P^{t} \widetilde{\mu}=\widetilde{\mu} \quad \text { for } t \in \mathbb{R}_{+} .
$$

On the other hand if $\widetilde{\mu} \in \mathcal{M}_{1}(Y)$ satisfies (3.4), then $\mu_{0}=H \widetilde{\mu}$ satisfies (3.3). 
Proof. Denote by $\left\{S^{t}\right\}_{t \geq 0}$ the semigroup of operators corresponding to the unperturbed system (2.4), i.e.

$$
S^{t} f(x, k)=f\left(\Pi_{k}(t, x), k\right) \quad \text { for } f \in C(Y),(x, k) \in Y .
$$

Denote by $\left\{U^{t}\right\}_{t \geq 0}$ the semigroup of operators corresponding to the stochastic differential equation $d u(t)=a(u(t), \xi(t)) d t$, that is,

$$
U^{t} f(x, k)=\sum_{s=1}^{N} f\left(\Pi_{s}(t, x), s\right) p_{k s}(x) .
$$

Just as $\left\{T^{t}\right\}_{t \geq 0}$, the families $\left\{S^{t}\right\}_{t \geq 0}$ and $\left\{U^{t}\right\}_{t \geq 0}$ are continuous semigroups of linear operators acting on $C(Y)$. For every continuously differentiable function $f$ with compact support, the infinitesimal generators $A$ of $\left\{U^{t}\right\}_{t \geq 0}$ and $A_{0}$ of $\left\{S^{t}\right\}_{t \geq 0}$ are given by

$$
\begin{aligned}
A f(x, k) & =\sum_{s=1}^{N}\left\langle a(x, s) \mid f_{x}(x, s)\right\rangle p_{k s}(x), \\
A_{0} f(x, k) & =\left\langle a(x, k) \mid f_{x}(x, k)\right\rangle,
\end{aligned}
$$

where $\langle\cdot \mid \cdot\rangle$ denotes the scalar product in $\mathbb{R}^{m}$.

Denote by $B$ the infinitesimal generator for the semigroup $\left\{T^{t}\right\}_{t \geq 0}$ on $C_{0}(Y)$. Since $C_{0}^{(1)}(Y) \subset D(B), B$ is given by

$$
\begin{aligned}
B f(x, k)= & \left\langle a(x, k) \mid f_{x}(x, k)\right\rangle-\lambda f(x, k) \\
& +\lambda \sum_{s=1}^{N} \int_{\Theta} f(q(x, \theta), s) p_{k s}(q(x, \theta)) d \nu(\theta)
\end{aligned}
$$

for $f \in C_{0}^{(1)}(Y)$. Thus

$$
B f=A_{0} f-\lambda f+\lambda Q W f
$$

where $Q: C(Y) \rightarrow C(Y)$ is the bounded linear operator given by

$$
Q f(x, k)=\int_{\Theta} f(q(x, \theta), k) d \nu(\theta) \quad \text { for } f \in C(Y) \text { and }(x, k) \in Y
$$

and

$$
W f(x, k)=\sum_{s=1}^{N} f(x, s) p_{k s}(x) \quad \text { for } f \in C(Y) \text { and }(x, k) \in Y .
$$

The domains $D(B), D(A)$ and $D\left(A_{0}\right)$ are identical.

Let $\mu_{0}$ be the invariant measure for $P$. Define $\widetilde{\mu}=G \mu_{0}$. Since

$$
R(\lambda, A) f(x, k)=\int_{0}^{\infty} e^{-\lambda t} U^{t} f(x, k) d t=\sum_{s=1}^{N} \int_{0}^{\infty} e^{-\lambda t} f\left(\Pi_{s}(t, x), s\right) p_{k s}(x) d t
$$


for $f \in C(Y)$, from (2.9), (3.7) and (3.10) we obtain

$$
\begin{array}{llrl}
P \mu & =\lambda Q^{*} R^{*}(\lambda, A) \mu, & & \mu \in \mathcal{M}_{1}(Y), \\
G \mu & =\lambda R^{*}(\lambda, A) \mu, & & \mu \in \mathcal{M}_{1}(Y),
\end{array}
$$

where $Q^{*}$ and $R^{*}(\lambda, A)$ are the operators adjoint to $Q$ and $R(\lambda, A)$. Hence

$$
\widetilde{\mu}=\lambda R^{*}(\lambda, A) Q^{*} \widetilde{\mu} .
$$

From this we have

$$
\langle f, \widetilde{\mu}\rangle=\langle\lambda Q R(\lambda, A) f, \widetilde{\mu}\rangle \quad \text { for } f \in C(Y) .
$$

Since $R(\lambda, A)=W R\left(\lambda, A_{0}\right)$ we obtain

$$
\langle f, \widetilde{\mu}\rangle=\left\langle\lambda Q W R\left(\lambda, A_{0}\right) f, \widetilde{\mu}\right\rangle \quad \text { for } f \in C(Y) .
$$

Substituting $f=\left(\lambda I-A_{0}\right) g$ we have

$$
\left\langle\lambda I-A_{0} g, \widetilde{\mu}\right\rangle=\langle\lambda Q W g, \widetilde{\mu}\rangle \quad \text { for } g \in C_{0}^{(1)}(Y),
$$

which according to $(3.9)$ reduces to

$$
\langle B g, \widetilde{\mu}\rangle=0 \quad \text { for } g \in D(B) .
$$

Now, since $T^{s} h \in D(B)$ for $h \in D(B)$ and

$$
T^{t} h-h=\int_{0}^{t} B T^{s} h d s \quad \text { for } h \in D(B),
$$

we obtain $\left\langle T^{t} h-h, \widetilde{\mu}\right\rangle=0$. This is equivalent to

$$
\left\langle h, P^{t} \widetilde{\mu}\right\rangle=\langle h, \widetilde{\mu}\rangle \quad \text { for } h \in D(B) .
$$

Since the set $D(B)$ is dense in $C(Y)$, this implies $P^{t} \widetilde{\mu}=\widetilde{\mu}$ for $t \in \mathbb{R}_{+}$.

Next, we show that if $\widetilde{\mu}$ is an invariant measure for the semigroup $\left\{P^{t}\right\}_{t>0}$ then $\mu_{0}=Q^{*} \widetilde{\mu}$ is an invariant measure for the operator $P$. From $P^{t} \widetilde{\mu}=\widetilde{\mu}$ it follows that

$$
\left\langle T^{t} g-g, \widetilde{\mu}\right\rangle=0 \quad \text { for } g \in C(Y) .
$$

Since $B$ is the infinitesimal generator of the semigroup $\left\{T^{t}\right\}_{t \geq 0}$, this implies

$$
\langle B g, \widetilde{\mu}\rangle=0 \quad \text { for } g \in D(B) .
$$

According to (3.9) this equality may be rewritten in the form

$$
\left\langle\left(\lambda I-A_{0}\right) g, \widetilde{\mu}\right\rangle=\langle\lambda Q W g, \widetilde{\mu}\rangle \quad \text { for } g \in D(B)=D\left(A_{0}\right) .
$$

Substituting $g=R\left(\lambda, A_{0}\right) f$ gives

$$
\langle f, \widetilde{\mu}\rangle=\left\langle\lambda Q W R\left(\lambda, A_{0}\right) f, \widetilde{\mu}\right\rangle=\langle\lambda Q R(\lambda, A) f, \widetilde{\mu}\rangle, \quad f \in C(Y) .
$$

This implies $\widetilde{\mu}=\lambda R^{*}(\lambda, A) Q^{*} \widetilde{\mu}$. From this we obtain immediately

$$
\mu_{0}=Q^{*} \widetilde{\mu}=\lambda Q^{*} R^{*}(\lambda, A) \mu_{0}=P \mu_{0} .
$$


By Theorem 3.1 the existence of an invariant measure for $P$ implies the existence of an invariant measure for the continuous time semigroup $\left\{P^{t}\right\}_{t \geq 0}$. In particular we have the following result.

THEOREM 3.2. Assume that there exist nonnegative constants $\alpha<1$ and $\beta$ such that

$$
\sum_{s=1}^{N} \int_{\Theta} \int_{0}^{\infty}\left\|q\left(\Pi_{s}(t, x), \theta\right)\right\| p_{k s}(x) \lambda e^{-\lambda t} d t d \nu(\theta) \leq \alpha\|x\|+\beta
$$

for $x \in \mathbb{R}^{m}, k \in\{1, \ldots, N\}$. Then $P$ has an invariant measure $\mu_{0}$ and $\widetilde{\mu}=G \mu_{0}$ is an invariant distribution with respect to $\left\{P^{t}\right\}_{t \geq 0}$.

Proof. The operator $U$ defined by (2.10) is a Feller operator. Thus it can be extended to all continuous nonnegative functions $f: Y \rightarrow Y$ by

$$
U f(x, k)=\lim _{n \rightarrow \infty} U f_{n}(x, k)
$$

where $\left\{f_{n}\right\}_{n \in \mathbb{N}} \subset C(Y)$ is an arbitrary increasing sequence converging pointwise to $f$. Evidently this extension is given by the same formula (2.10). Thus setting $h(x, k)=\|x\|$ we may rewrite inequality (3.12) in the form

$$
U h(x, k) \leq \alpha h(x, k)+\beta \quad \text { for } x \in \mathbb{R}^{m}, k \in\{1, \ldots, N\} .
$$

According to Proposition 7.1 of [11] this implies the existence of an invariant measure $\mu_{0}$ for $P$. A straightforward application of Theorem 3.1 completes the proof.

Remark 3.3. Assume that the solutions $\Pi_{k}: \mathbb{R}_{+} \times \mathbb{R}^{m} \rightarrow \mathbb{R}^{m}$ of the equations (2.4) and the transition probabilities $p_{k s}: \mathbb{R}^{m} \rightarrow[0,1]$ satisfy

$$
\sum_{k=1}^{N} p_{i k}(y)\left\|\Pi_{k}(t, x)-\Pi_{k}(t, y)\right\| \leq L e^{-\eta t}\|x-y\|
$$

for $x, y \in \mathbb{R}^{m}$ and $i=1, \ldots, N$ and the function $q: \mathbb{R}^{m} \times \Theta \rightarrow \mathbb{R}^{m}$ satisfies

$$
\sup _{t \geq 0} \int_{\Theta}\left\|q\left(\Pi_{s}(t, 0), \theta\right)\right\| d \nu(\theta)<\infty \quad \text { for } s=1, \ldots, N .
$$

If in addition the positive constants $\eta, \lambda, L, L_{q}$ satisfy

$$
L L_{q}-\eta / \lambda<1
$$

then the condition (3.12) of Theorem 3.2 is satisfied.

4. The generalized Rényi dimension of invariant measures. Theorem 3.1 allows us to evaluate the generalized Rényi dimension of the measure $\widetilde{\mu}$ if the dimension of $\mu_{0}$ is known and vice versa.

We use the Lévy concentration function to calculate the dimension of a probability measure [9]. Given $\mu \in \mathcal{M}_{1}(Y)$ we define the Lévy concentration 
function $Q_{\mu}: \mathbb{R}_{+} \rightarrow \mathbb{R}_{+}$by

$$
Q_{\mu}(r)=\sup \{\mu(K(x, r)): x \in Y\} .
$$

Then

$$
\overline{\operatorname{dim}}_{\infty} \mu=\limsup _{r \rightarrow 0} \frac{\log Q_{\mu}(r)}{\log r}, \quad \underline{\operatorname{dim}}_{\infty} \mu=\liminf _{r \rightarrow 0} \frac{\log Q_{\mu}(r)}{\log r} .
$$

The dimension $\operatorname{dim}_{\infty} \mu$ has two important properties. First, it is relatively easy to calculate. Second, it is strongly related to the Hausdorff dimension of sets.

Recall that the Hausdorff dimension of a measure $\mu \in \mathcal{M}_{1}(Y)$ is given by

$$
\operatorname{dim}_{\mathrm{H}} \mu=\inf \left\{\operatorname{dim}_{\mathrm{H}} A: A \in \mathcal{B}(Y), \mu(A)=1\right\}
$$

where $\operatorname{dim}_{\mathrm{H}} A$ is the Hausdorff dimension of the set $A$.

It can be easily proved [9] that

Proposition 4.1. For every $\mu \in \mathcal{M}_{1}(Y)$ we have

$$
\operatorname{dim}_{\mathrm{H}} \operatorname{supp} \mu \geq \underline{\operatorname{dim}}_{\infty} \mu \text {. }
$$

We are going to prove only some results concerning the generalized Rényi dimensions.

We will assume that the solutions $\Pi_{k}: \mathbb{R} \times \mathbb{R}^{m} \rightarrow \mathbb{R}^{m}$ of equations (2.4) satisfy

$$
\left\|\Pi_{k}(-t, x)-\Pi_{k}(-t, y)\right\| \leq c_{k} e^{\beta t}\|x-y\|
$$

for $x, y \in \mathbb{R}^{m}, k \in\{1, \ldots, N\}$ and $t \geq 0$, where $\beta \in \mathbb{R}$ and $c_{k}>0$ are constants. Since for every fixed $k \in\{1, \ldots, N\}$ the coefficient $a(x, k)$ is Lipschitzean this inequality is automatically satisfied for sufficiently large $\beta$.

THeOREM 4.1. If $\lambda>\beta \underline{\operatorname{dim}}_{\infty} \mu_{0}$ then

$$
\underline{\operatorname{dim}}_{\infty} \tilde{\mu} \geq \underline{\operatorname{dim}}_{\infty} \mu_{0} .
$$

Proof. Fix $h<\underline{\operatorname{dim}}_{\infty} \mu_{0}$ such that $\lambda>\beta h$. From the definition of $\underline{\operatorname{dim}}_{\infty} \mu_{0}$ it follows that there is $0<r_{0}<b$ (where $b=\varrho_{0}(k, l)$ for $k \neq l$ ) such that

$$
Q_{\mu_{0}}(r) \leq r^{h} \quad \text { for } r \in\left(0, r_{0}\right) .
$$

Since $\widetilde{\mu}=G \mu_{0}$ where

$$
G \mu(A)=\sum_{s=1}^{N} \int_{Y} \int_{0}^{\infty} 1_{A}\left(\Pi_{s}(t, x), s\right) p_{k s}(x) \lambda e^{-\lambda t} d t d \mu(x, k) \quad \text { for } A \in \mathcal{B}(Y)
$$

we have

$$
\begin{aligned}
& \tilde{\mu}(K((\bar{x}, \bar{k}), r)) \\
& \quad=\int_{0}^{\infty} \int_{\mathbb{R}^{m} \times\{1, \ldots, N\}} 1_{K((\bar{x}, \bar{k}), r)}\left(\Pi_{\bar{k}}(t, x), \bar{k}\right) \lambda e^{-\lambda t} p_{k \bar{k}}(x) d t d \mu_{0}(x, k) .
\end{aligned}
$$


Set

$$
\gamma_{s}=\sup \left\{p_{k s}(x):(x, k) \in \mathbb{R}^{m} \times\{1, \ldots, N\}\right\} .
$$

Then we obtain

$$
\widetilde{\mu}(K((\bar{x}, \bar{k}), r)) \leq \gamma_{\bar{k}} \int_{0}^{\infty} \mu_{0}\left(\Pi_{\bar{k}}(-t, K(\bar{x}, r)) \times\{1, \ldots, N\}\right) \lambda e^{-\lambda t} d t .
$$

Consequently, by (4.2) we have

$$
\Pi_{\bar{k}}(-t, K(\bar{x}, r)) \subset K\left(\Pi_{\bar{k}}(-t, \bar{x}), c_{\bar{k}} r e^{\beta t}\right) .
$$

Thus

$$
\widetilde{\mu}(K((\bar{x}, \bar{k}), r)) \leq \gamma_{\bar{k}} N \int_{0}^{\infty} Q_{\mu_{0}}\left(c_{\bar{k}} r e^{\beta t}\right) \lambda e^{-\lambda t} d t
$$

Let

$$
r<\min \left\{r_{0}, r_{0} / \max _{k} c_{k}\right\}
$$

Consider first the case $\beta>0$ and define $T\left(r_{\bar{k}}\right)=\beta^{-1} \log \left(r_{0} / c_{\bar{k}} r\right)$. Then

$\widetilde{\mu}(K((\bar{x}, \bar{k}), r)) \leq \gamma_{\bar{k}} N\left(\int_{0}^{T\left(r_{\bar{k}}\right)} Q_{\mu_{0}}\left(c_{\bar{k}} r e^{\beta t}\right) \lambda e^{-\lambda t} d t+\int_{T\left(r_{\bar{k}}\right)}^{\infty} Q_{\mu_{0}}\left(c_{\bar{k}} r e^{\beta t}\right) \lambda e^{-\lambda t} d t\right)$.

To estimate the first integral we may use inequality (4.4). Then an elementary calculation gives

$$
\widetilde{\mu}(K((\bar{x}, \bar{k}), r)) \leq\left(\gamma_{\bar{k}} N \frac{\lambda}{\lambda-\beta h}\left(c_{\bar{k}}\right)^{h}\right) r^{h}+\gamma_{\bar{k}} N \int_{T\left(r_{\bar{k}}\right)}^{\infty} Q_{\mu_{0}}\left(c_{\bar{k}} r e^{\beta t}\right) \lambda e^{-\lambda t} d t
$$

Since $c_{\bar{k}} r<r_{0}$ and $\lambda>\beta h$ we obtain

$$
\widetilde{\mu}(K((\bar{x}, \bar{k}), r)) \leq C r^{h} \quad \text { for } r<\min \left\{r_{0}, r_{0} / \max _{k} c_{k}\right\}
$$

where

$$
C=\frac{\lambda N \max _{k}\left(c_{k}\right)^{h} \gamma_{k}}{\lambda-\beta h}+\frac{N \max _{k}\left(c_{k}\right)^{h} \gamma_{k}}{r_{0}^{h}}
$$

Since the inequality (4.6) is satisfied for every $(\bar{x}, \bar{k}) \in \mathbb{R}^{m} \times\{1, \ldots, N\}$, according to the definition of $Q_{\widetilde{\mu}}(r)$ we obtain

$$
Q_{\widetilde{\mu}}(r) \leq C r^{h} \text {. }
$$

If $\beta \leq 0$ the calculation is even simpler and gives (4.7) with

$$
C=\frac{\lambda N \max _{k}\left(c_{k}\right)^{h} \gamma_{k}}{\lambda-\beta h} .
$$

From inequality (4.7) it follows that $\underline{\operatorname{dim}}_{\infty} \widetilde{\mu} \geq h$. Passing to the limit as $h \rightarrow \underline{\operatorname{dim}}_{\infty} \mu_{0}$ we obtain (4.3). 
Define

$$
L(\theta)=\inf \left\{\frac{\|q(x, \theta)-q(y, \theta)\|}{\|x-y\|}: x \neq y\right\} .
$$

THEOREM 4.2. Assume that

$$
L_{0}=\underset{\theta \in \Theta}{\operatorname{essinf}} L(\theta)>0 .
$$

Then

$$
\underline{\operatorname{dim}}_{\infty} \widetilde{\mu} \leq \underline{\operatorname{dim}}_{\infty} \mu_{0} \quad \text { and } \quad \overline{\operatorname{dim}}_{\infty} \widetilde{\mu} \leq \overline{\operatorname{dim}}_{\infty} \mu_{0}
$$

Proof. From (4.8) and (4,9) it follows that almost everywhere with respect to $\nu$,

$$
\operatorname{diam}\{(x, k):(q(x, \theta), k) \in K((\bar{x}, \bar{k}), r)\} \leq 2 r L_{0}^{-1}
$$

for every $(\bar{x}, \bar{k}) \in \mathbb{R}^{m} \times\{1, \ldots, N\}$ and $0<r<b$. Thus according to the definition of $Q_{\widetilde{\mu}}$,

$$
\widetilde{\mu}(\{(x, k):(q(x, \theta), k) \in K((\bar{x}, \bar{k}), r)\}) \leq Q_{\widetilde{\mu}}\left(2 r L_{0}^{-1}\right) .
$$

Since $\mu_{0}=H \widetilde{\mu}$ where

$$
H \mu(A)=\int_{Y} \int_{\Theta} 1_{A}(q(x, \theta), k) d \nu(\theta) d \mu(x, k),
$$

we obtain

$$
\mu_{0}(K((\bar{x}, \bar{k}), r))=\int_{\Theta} \widetilde{\mu}(\{(x, k):(q(x, \theta), k) \in K((\bar{x}, \bar{k}), r)\}) d \nu(\theta) .
$$

From (4.11) we have $\mu_{0}(K((\bar{x}, \bar{k}), r)) \leq Q_{\widetilde{\mu}}\left(2 r L_{0}^{-1}\right)$ for $(\bar{x}, \bar{k}) \in \mathbb{R}^{m} \times$ $\{1, \ldots, N\}$ and $0<r<b$. Consequently, $Q_{\mu_{0}}(r) \leq Q_{\widetilde{\mu}}\left(2 r L_{0}^{-1}\right)$, which implies (4.10).

\section{References}

[1] O. Diekmann, H. J. A. M. Heijmans and H. R. Thieme, On the stability of the cell size distribution, J. Math. Biol. 19 (1984), 227-248.

[2] R. Fortet et B. Mourier, Convergence de la répartition empirique vers la répartition théorétique, Ann. Sci. École Norm. Sup. 70 (1953), 267-285.

[3] I. L. Gikhman and A. Y. Skorokhod, Stochastic Differential Equations and Their Applications, Naukova Dumka, Kiev, 1982 (in Russian).

[4] K. Horbacz, Randomly connected differential equations with Poisson type perturbations, to appear.

[5] K. Horbacz and T. Szarek, Randomly connected dynamical systems on Banach spaces, Stochastic Anal. Appl. 19 (2001), 519-543.

[6] A. Lasota, From fractals to stochastic differential equations, in: Chaos- the Interplay between Stochastic and Deterministic Behaviour (Karpacz, 1995), Springer, 1995, 235-255. 
[7] A. Lasota and M. C. Mackey, Noise and statistical periodicity, Phys. D 28 (1987), $143-154$.

[8] —, Chaos, Fractals and Noise-Stochastic Aspects of Dynamics, Springer, New York, 1994.

[9] A. Lasota and J. Myjak, Concentration dimension of measures, Bull. Polish Acad. Sci. Math., to appear.

[10] A. Lasota and J. Traple, Invariant measures related with Poisson driven stochastic differential equations, to appear.

[11] A. Lasota and J. A. Yorke, Lower bound technique for Markov operators and iterated function systems, Random Comput. Dynamics 2 (1994), 41-77.

[12] K. S. Lau and S. M. Ngai, Multifractal measures and a weak separation condition, Adv. Math. 141 (1999), 45-96.

[13] L. Olsen, A multifractal formalism, ibid. 116 (1995), 82-195.

[14] K. Pichór and R. Rudnicki, Continuous Markov semigroups and stability of transport equations, J. Math. Anal. Appl. 249 (2000), 668-685.

[15] J. Traple, Markov semigroups generated by Poisson driven differential equations, Bull. Polish Acad. Sci. Math. 44 (1996), 161-182.

Institute of Mathematics

Silesian University

40-007 Katowice, Poland

E-mail: horbacz@ux2.math.us.edu.pl

Reçu par la Rédaction le 4.5.2001

Révisé le 19.11.2001 